\title{
Pregnancy-related changes in bone turnover markers are not accompanied by mineral mobilisation in the appendicular skeleton in women with habitually low calcium intake
}

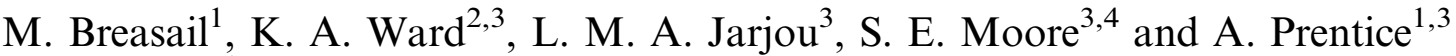 \\ ${ }^{1}$ MRC Nutrition and Bone Health Research Group, Cambridge, UK, \\ ${ }^{2}$ MRC Lifecourse Epidemiology Unit, University of Southampton, Southampton, UK, \\ ${ }^{3}$ MRC Unit The Gambia, London School of Hygiene and Tropical Medicine, Banjul, Gambia and \\ ${ }^{4}$ Department of Women and Children's Health, King's College London, London, UK
}

In pregnancy, changes in maternal calcium $(\mathrm{Ca})$ economy occur to satisfy fetal $\mathrm{Ca}$ demand ${ }^{(1)}$. Whether maternal mineral reserves facilitate these requirements in a population with habitually low Ca intakes $(\sim 400 \mathrm{mg} / \mathrm{day})^{(2)}$ is difficult to quantify and no data exist from sub-Saharan Africa. Our aim was to determine whether changes in maternal bone metabolism occurred in pregnancy using bone turnover markers (BTMs) and peripheral quantitative computed tomography (pQCT) between early second and third trimesters.

Pregnant rural Gambians aged 18-45 years $(n=467)$ participating in a trial of antenatal nutritional supplements (ISRCTN49285450) $^{(3)}$ were invited for longitudinal measurements: biochemistry including maternal BTMs (serum collagen type 1 cross-linked $\beta$-C-telopeptide (CTX), type 1 procollagen N-terminal (P1NP) plus parathyroid hormone (PTH) and 1,25(OH)2D)) and pQCT scans at the non-dominant radius and tibia. pQCT measures were total volumetric bone mineral density (vBMD), trabecular vBMD, total area at distal sites $(4 \%)$ and cortical vBMD, bone mineral content (BMC), total area at proximal sites $(33 / 38 \%)$. Supplements were iron folic acid (IFA; standard care), multiple micronutrients (MMN), protein energy (PE), PE + MMN. Independent $\mathrm{t}$-tests tested whether within-group and pooled change differed significantly from 0 . Multiple regression adjusting for age, and then supplement group tested if between-visit change differed by group. Data for change are expressed as mean [95CI $\%$.

Visits occurred at mean (SD) 14(3) and 31(1) weeks of pregnancy. Pregnancy-related changes in BTMs were observed with CTX increasing by $23.0[15.09,29.29] \%$ and P1NP decreasing by $32.4[-37.19,-28.17] \%$. Changes in maternal PTH and $1,25(\mathrm{OH}) 2 \mathrm{D}$ were also evident, increasing by $13.2[8.44,19.34] \%$ and $21.0[17.67,24.29] \%$ respectively. No significant group differences were seen. Radial trabecular and cortical vBMD, and BMC increased between visits by $1.15[0.55,1.75] \%, 0.35[0.26,0.44]^{\%}$ and $0.55[0.41,0.68]^{\%} \%$ respectively. At the tibia, total and trabecular vBMD increased by $0.34[0.15,0.54] \%$ and $0.46[0.17,0.74] \%$, while cortical vBMD and BMC increased by $0.41[0.24,0.58] \%$, and $0.47[0.25,0.69] \%$ respectively. At the radius change in cortical vBMD in MMN and $\mathrm{PE}$ was $0.54[-1.01,-0.07] \%$ and $0.73[-1.20,-0.26] \%$ lower than IFA. Increase in radius cortical CSA in MMN was 0.67 $[0.10,1.25] \%$ greater than IFA. Change in tibia total vBMD was $0.76[0.22,1.30] \%$ greater in PE + MNN vs IFA.

Between-visit changes in BTMs suggest pregnancy-related changes in maternal bone mineral homeostasis. However, no evidence of bone mineral mobilisation was observed in the peripheral skeleton, rather modest increases were observed. No consistent differences were found by supplement group, despite the MMN groups containing vitamin D. In this population, with habitually low Ca intakes, the peripheral skeleton is not mobilised as a Ca source for the offspring.

However, in light of the large changes in BTMs it is possible that mobilisation was occurring at axial sites because BTMs in the circulation reflect changes throughout the skeleton.

\section{References}

1. Prentice, A. (2003) J Nutr, $133(5$ Suppl 2): p. 1693s-1699s.

2. Prentice, A. (1994) Adv Exp Med Biol, 352: p. 243-55.

3. Moore, S.E., et al. (2012) BMC Pregnancy Childbirth, 12: p. 107. 